\title{
Influencia de la actividad física sobre la resiliencia en adultos con dolor de hombro
}

\section{Influence of physical activity on resilience in adults with shoulder pain}

\author{
Félix Zurita Ortega ${ }^{1 *}$, Tamara Espejo Garcés ${ }^{2}$, Cristian Cofré Boladós ${ }^{3}$, \\ Asunción Martínez Martínez ${ }^{4}$, Manuel Castro Sánchez ${ }^{2}$ y Ramón Chacón Cuberos ${ }^{2}$
1 Doctor del Área de Corporal de la Universidad de Granada (España). 2 Grupo de Investigación HUM-238 de la Universidad de Granada (España). 3 Doctor por la Universidad Santo Tomás de Santiago de Chile (Chile). 4 Doctora en Métodos y de Investigación y Diagnostico en Educación de la Universidad de Granada (España).

\begin{abstract}
Resumen: La calidad de vida de la población adulta chilena está aumentando, lo que genera un gran interés por los estamentos sanitarios-educativos. Este trabajo pretende estudiar la relación que tiene la práctica de actividad física en la resiliencia de adultos mayores con problemas de hombro. Se realizó un estudio de carácter descriptivo y de corte transversal en 41 adultos de Santiago de Chile (Chile), con una edad media de 58.17 ańos (DT=11.754), utilizándose como principales instrumentos el cuestionario CD-RISC y la Escala de evaluación de hombro "UCLA. Los resultados arrojaron que la mitad de los adultos realizan actividad física, obteniendo un locus de control y compromiso aceptable, siendo la espiritualidad menos valorada. No se halló relación entre la práctica de actividad física y el género, pero si con las dimensiones de resiliencia con niveles elevados, poniendo de manifiesto que hacer deporte aumenta la resiliencia en adultos.

Palabras Clave: Actividad Física, Deporte, Adultez, Resiliencia
\end{abstract}

Abstract: The quality of life of Chilean adult population is increasing, which generates a great interest in the health-educational establishments. This research aims to study the relationship between physical activity and resilience in older adults with pathologies of shoulder. It was conducted a descriptive and cross-sectional study in 41 adults from Santiago of Chile, with an average age of 58.17 years old $(\mathrm{SD}=11.754)$, using as a main instruments the CD-RISC questionnaire and the scale of shoulder' assessment "UCLA". Results showed that half of adults do physical activity and that they show an acceptable locus of control and compromise, being spirituality the least scored. Physical activity and gender did not show relations, although the dimensions of resilience with high score were related to sport practice.

Key Words: Physical activity, Sport, Adulthood, Resilence.

\section{Introducción}

En la sociedad actual el estudio de los beneficios que tiene la práctica de actividad física sobre el estado de salud en personas adultas es innegable (Canellas y Rovira, 1995; JiménezBeatty, Graupera, Martínez del Castillo, Martín y Campos, 2007; Martínez, Jiménez-Beatty, González, Graupera, Martín, Campos y Del Hierro, 2009; Zurita, Fernández, Cepero, Zagalaz, Valverde y Ramírez, 2009; López-Sánchez, LópezSánchez y Díaz, 2015), repercutiendo en un incremento del bienestar físico, mental y social, y con el consiguiente aumento de su estado de salud (Azpiazu, Jentoft, Villagrasa, Abanades, García y Alvear, 2002; Tomás, Meléndez y Navarro, 2008; Vivaldi y Barra, 2012).

Si bien también debemos de tener en cuenta lo planteado por Rioseco, Quezada, Ducci y Torres (2008), sobre ciertas experiencias (viudez, jubilación, cambios de residencia, menor participación en la sociedad y mayor vulnerabilidad ante acontecimientos inesperados), que pueden hacer que el adulto se sienta realizado o no, en el otro extremo de condicionar la generalización de su estado de bienestar. El ejercicio físico

Dirección para correspondencia [Correspodence address]: Félix Zurita Ortega. Facultad de Ciencias de la Educación. Campus de Cartuja s/n. 18071 Granada (Espańa). E-mail: felixzo@ugr.es moderado va a aportar beneficios al estado de salud, aunque en ocasiones puede comportar lesiones al aparato locomotor y un riesgo cardiovascular, el beneficio supera al riesgo ( $\mathrm{Su}$ birats, Subirats y Soteras, 2012). Algunos colectivos de personas mayores (mujeres) en ocasiones reportan menores niveles de bienestar, con aumento de los niveles de agotamiento y por ende una menor actividad física (Hicks, Epperly y Barnes, 2001; Pinguart y Sorensen, 2001).

Las lesiones de hombro y su estudio han estado casi siempre asociadas al deporte de alta competición (Peixoto, Fiquetti, Polisello, Barbosa, Ejnisman y Cohen, 2013).En este sentido, en una población adulta que tiene otras prioridades de tipo familiar o laboral, es necesario prepararlapara afrontar y prevenir posibles anormalidades de hombro que pueden llegar a producirse en años posteriores, siendo muy escasos los estudios realizados sobre la relación entre patología de hombro y actividad física en adultos (Zurita et al., 2009).

Al hilo de todo esto y basándose en las aportaciones de Haase (2004); Vinson (2008) o Anthony, Alter y Jenson (2009), la resiliencia es una capacidad que ha mostrado su efectividad en diversas intervenciones de diversa índole, puesto que promueve el proceso de superar los efectos negativos 
de la exposición al riesgo, el afrontamiento exitoso de las experiencias estresantes, y la evitación de las trayectorias negativas asociadas al riesgo.

Ante esta situación se entiende que la actividad física se encuentra bastante asociada a la capacidad resiliente (CastroSánchez, Chacón-Cuberos, Zurita y Espejo, 2016; ChacónCuberos, Castro, Espejo y Zurita, 2016), si bien ha sido poco tratada en el ámbito de las personas adultas.

Los profesionales del deporte, profesores o psicólogos son los máximos responsables de cómo se realizan los diversos programas de intervención y la detección de cualquier anormalidad a nivel físico y psicológico por lo que este trabajo intenta ir un paso más allá y estudiar de manera más explícitala relación positiva que tiene la realización de actividad física sobre factores psicológicos y de evaluación en adultos con problemas de hombro.

Los objetivos del trabajo son determinarla prevalencia de actividad física, resiliencia y de estado de hombro en adultos de Santiago de Chile, y determinar la influencia de práctica de actividad física en la capacidad de resiliencia y en sus diversas dimensiones así como en el estado en que se encuentra el hombro.

\section{Material y métodos}

\section{a) Diseño y participantes}

Se trata de un estudio observacional, descriptivo y de corte transversal. La selección de la muestra se realizó por muestreo de conveniencia sobre 261 sujetos de entre 45 y 65 ańos con patología de hombro, se les solicitó permiso para la participación en este estudio, de los cuales respondieron 51 afirmativamente, finalmente participaron en esta investigación un total de 41 adultos mayores de 50 a 65 ańos ( $\mathrm{X}=58.17$ ańos; $\mathrm{DT}=11.754)$, que acudían regularmente al centro de ejercicio adaptado de la asociación cristiana de jóvenes de Santiago de Chile. De los participantes, 19 (46,3\%) eran hombres, mientras los restantes $22(53,7 \%)$ eran mujeres.

\section{b) Instrumentos de medida}

Se emplearon diversos instrumentos validados para evaluar la patología de hombro, capacidad de resiliencia y actividad físico-deportiva.

- El Cuestionario de Autoregistro y Sociodemográfico, registraba la edad, género, práctica de actividad física (categorizado en nunca, a veces, he practicado, pero hace tres años que no y siempre), modalidad realizada (categorizado en no practica, ejercicio de mantenimiento, ciclismo, yoga-taichi y fútbol) y tipología de afección de hombro.

- El test "Connor-DavidsonResilienceScale", que fue propuesto por Connor y Davidson (2003), está formado por 25 ítems, y nos determina la capacidad de Resiliencia, se valora mediante una escala Likert de cuatro opciones de respuesta, que va desde el 1 que corresponde con "Nada de Acuerdo" hasta el 4 a "Totalmente de Acuerdo". Utilizado en el contexto deportivo por Gucciardi, Jackson, Coulter y Mallett (2011) en su estudio de jugadores de Criqueten Australia, en el contexto sanitario Lamond, Depp, Allison, Langer, Reichstadt y Moore (2008)establecen la importancia de la resiliencia en mujeres mayores con principio de déficit cognitivo. Se divide en cinco dimensiones: locus de control y compromiso, desafío de conducta orientada a la acción, autoeficacia y resistencia al malestar, optimismo y adaptación a situaciones estresantes y espiritualidad.

Su fiabilidad en este estudio presenta un alpha de Cronbach de $\alpha=.975$, por dimensiones se obtuvieron los siguientes datos: locus de control y compromiso con un alpha de Cronbach de $\alpha=.936$; desafío de conducta orientada a la acción $(\alpha=.608)$, autoeficacia y resistencia al malestar $(\alpha=.950)$, optimismo y adaptación a situaciones estresantes $(\alpha=.848)$ y en espiritualidad $(\alpha=.700)$, lo que establece un nivel alto de fiabilidad.

- Escala de evaluación de hombro "UCLA", este instrumento recogido de su versión original por Amstutz, Sew y Clarke (1981), en la Universidad de los Ángeles. Esta escala ha sido la más empleada a nivel mundial puesto que clasifica los hombros mediante un sistema de puntuación, donde establece cinco elementos de valoración en base al dolor, función, flexión activa, fuerza muscular a la flexión activa y satisfacción; mediante el sumatorio de cada una de las categorías, se determina una puntuación global: excelente (entre 34-35 puntos), bueno (entre 28-33 puntos), regular (entre 21-27 puntos) y malo (entre 0-20 puntos).

\section{c) Procedimiento}

Se solicitó la colaboración de algunos centros deportivos y rehabilitadores de Santiago de Chile para participar en la investigación exponiéndoles de forma breve el objetivo del estudio y solicitando la colaboración de sus participantes; asimismo se adjuntó un modelo de consentimiento informado para que fuese rellenado por ellos mismos. La administración de las pruebas se realizó durante los meses de noviembre y diciembre de 2015, siguiendo los pasos del protocolo de aplicación, indicándoles el total anonimato de las respuestas. Los encuestadores (médicos, fisioterapeutas y profesor de Educación Física) estuvieron presentes durante la recogida de los datos para confirmar la correcta realización de las pruebas o test. 
La recogida se desarrolló sin ningún tipo de anormalidad y finalizada los participantes volvían a su rutina de actividad. Al terminarse agradecía a los responsables su colaboración y se les informaba de la recepción en un futuro del informe de los resultados obtenidos al final del estudio.

\section{d)Análisis estadístico}

Se realizó mediante la utilización del Software SPSS 22.0 para Windows. El índice de participación fue del 81.20\% con un total de 3 cuestionarios invalidados por no acudir el día de la recogida de datos o por no cumplimentar correctamente las pruebas. Las técnicas de análisis utilizadas fueron de tipo descriptivo (frecuencias y medias), tablas de contingencia mediante la prueba de chi cuadrado y ANOVA.

\section{Resultados}

Se detectó que 23 (56.1\%) participantes realizaban actividad física de manera habitual, mientras que los restantes 18 adultos no practicaban. Asimismo los sujetos con evaluación mala según la prueba de hombro UCLA eran el 65,9\% de los casos, y entre las dimensiones de la resiliencia el locus de control y compromiso es el más puntuado $(\mathrm{M}=2.90)$, mientras que la espiritualidad es la menor valorada, como puede desprenderse de las cifras de la siguiente tabla. Tabla 1.

Tabla 1. Distribución de los valores descriptivos de la muestra.

\begin{tabular}{|c|c|c|}
\hline \multirow{2}{*}{ Género } & Masculino & $19(46.3 \%)$ \\
\hline & Femenino & $22(53.7 \%)$ \\
\hline \multirow{2}{*}{ Práctica Actividad Física } & Si Practica & $23(56.1 \%)$ \\
\hline & No Practica & $18(43.9 \%)$ \\
\hline \multirow{4}{*}{ Evaluación Hombro } & Excelente & $2(4.9 \%)$ \\
\hline & Bueno & $5(12.2 \%)$ \\
\hline & Regular & $7(17.1 \%)$ \\
\hline & Malo & $27(65.9 \%)$ \\
\hline \multirow{5}{*}{$\begin{array}{c}\text { Resiliencia } \\
\mathrm{M}=2.72(\mathrm{DT}=.741)\end{array}$} & Locus de control y compromiso & $\mathrm{M}=2.90(\mathrm{DT}=.825)$ \\
\hline & Desafío de conducta orientada a la acción & $\mathrm{M}=2.67(\mathrm{DT}=.818)$ \\
\hline & Autoeficacia y resistencia al malestar & $\mathrm{M}=2.77(\mathrm{DT}=.798)$ \\
\hline & Optimismo y adaptación a situaciones estresantes & $\mathrm{M}=2.70(\mathrm{DT}=.726)$ \\
\hline & Espiritualidad & $\mathrm{M}=2.55(\mathrm{DT}=.783)$ \\
\hline
\end{tabular}

La tabla 2, estudia en primer lugar la relación entre los que practican actividad física y el género no hallándose diferencias estadísticamente significativas ( $\mathrm{p}=.054)$. Los resultados arrojan la existencia de asociación entre la resiliencia y la práctica en todas las dimensiones $(p<0.005)$,excepto en la espiritualidad, dicha asociación viene establecida en todos los casos por los valores medios de aquellos sujetos que realizan actividad física que se encuentran incrementados respecto a los que no practican.
También se hallaron diferencias al comparar la evaluación del hombro con la realización de actividad física $(\mathrm{p}=.000)$, de esta forma se aprecia como en aquellos sujetos que hacen deporte la evaluación excelente, buena y regular esta incrementada con respecto a los sedentarios, que a su vez obtienen porcentajes más elevados en evaluación mala (88.8\%) que los activos (47.8\%). 
Tabla 2. Relación de la práctica de actividad física según género, resiliencia y evaluación

\begin{tabular}{|c|c|c|c|c|}
\hline & \multirow{2}{*}{$\begin{array}{l}\text { Variables Práctica de Actividad Física } \\
\text { Si Practicas }\end{array}$} & \multicolumn{2}{|c|}{ Frecuencias y Porcentajes } & \multirow{2}{*}{ Chi-Cuadrado $(\mathrm{p}=)$} \\
\hline & & No Practicas & & \\
\hline \multirow{2}{*}{ Género } & Masculino & $9(39.1 \%)$ & $10(55.6 \%)$ & \multirow{2}{*}{$\mathrm{p}=.054$} \\
\hline & Femenino & $14(60.9 \%)$ & $8(44.4 \%)$ & \\
\hline \multirow{6}{*}{ Resiliencia } & Resiliencia General & $\mathrm{M}=3.02(\mathrm{DT}=.682)$ & $\mathrm{M}=2.34(\mathrm{DT}=.649)$ & $10.380(\mathrm{p}=.003)^{* * *}$ \\
\hline & Locus de control y compromiso & $\mathrm{M}=3.35(\mathrm{DT}=.699)$ & $\mathrm{M}=2.34(\mathrm{DT}=.613)$ & $23.156(\mathrm{p}=.000)^{* * *}$ \\
\hline & Desafío de conducta orientada a la acción & $\mathrm{M}=2.89(\mathrm{DT}=.839)$ & $\mathrm{M}=2.39(\mathrm{DT}=.719)$ & $4.098(\mathrm{p}=.050)^{* * *}$ \\
\hline & Autoeficacia y resistencia al malestar & $\mathrm{M}=3.13(\mathrm{DT}=.737)$ & $\mathrm{M}=2.33(\mathrm{DT}=.644)$ & $13.350(\mathrm{p}=.001)^{* * *}$ \\
\hline & Optimismo y adaptación a situaciones estresantes & $\mathrm{M}=2.97(\mathrm{DT}=.648)$ & $\mathrm{M}=2.37(\mathrm{DT}=.694)$ & $8.344(\mathrm{p}=.006)^{* * *}$ \\
\hline & $\begin{array}{l}\text { Espiritualidad } \\
\end{array}$ & $\mathrm{M}=2.75(\mathrm{DT}=.760)$ & $\mathrm{M}=2.30(\mathrm{DT}=.757)$ & $3.666(\mathrm{p}=.063)$ \\
\hline \multirow{4}{*}{$\begin{array}{l}\text { Evaluación } \\
\text { UCLA }\end{array}$} & Excelente & $2(8.7 \%)$ & $0(0.0 \%)$ & \multirow{4}{*}{$\mathrm{p}=.000^{* * *}$} \\
\hline & Bueno & $4(17.4 \%)$ & $1(5.6 \%)$ & \\
\hline & Regular & $6(26.1 \%)$ & $1(5.6 \%)$ & \\
\hline & Malo & $11(47.8 \%)$ & $16(88.8 \%)$ & \\
\hline
\end{tabular}

En la tabla 3 se presentan los resultados de la comparativa entre el género y la resiliencia y evaluación UCLA, no hallán- dose relación estadísticamente significativa $(p<0.005)$ como puede apreciarse en la siguiente tabla. Tabla 3.

Tabla 3. Relación del género según resiliencia y evaluación.

\begin{tabular}{|c|c|c|c|c|}
\hline & \multirow{2}{*}{$\begin{array}{c}\text { Variables Género } \\
\text { Masculino }\end{array}$} & \multicolumn{2}{|c|}{ Frecuencias y Porcentajes } & \multirow[t]{2}{*}{ Chi-Cuadrado $(\mathrm{p}=)$} \\
\hline & & Femenino & & \\
\hline \multirow{6}{*}{ Resiliencia } & Resiliencia General & $\mathrm{M}=2.78(\mathrm{DT}=.624)$ & $\mathrm{M}=2.66(\mathrm{DT}=.839)$ & $.263(\mathrm{p}=.611)$ \\
\hline & Locus de control y compromiso & $\mathrm{M}=2.91(\mathrm{DT}=.746)$ & $\mathrm{M}=2.90(\mathrm{DT}=.906)$ & $.004(\mathrm{p}=.952)$ \\
\hline & Desafío de conducta orientada a la acción & $\mathrm{M}=2.73(\mathrm{DT}=.752)$ & $\mathrm{M}=2.61(\mathrm{DT}=.885)$ & $.226(\mathrm{p}=.637)$ \\
\hline & Autoeficacia y resistencia al malestar & $\mathrm{M}=2.82(\mathrm{DT}=.674)$ & $\mathrm{M}=2.73(\mathrm{DT}=.905)$ & $.127(\mathrm{p}=.724)$ \\
\hline & Optimismo y adaptación a situaciones estresantes & $\mathrm{M}=2.81(\mathrm{DT}=.623)$ & $\mathrm{M}=2.61(\mathrm{DT}=.809)$ & $.709(\mathrm{p}=.405)$ \\
\hline & $\begin{array}{c}\text { Espiritualidad } \\
\end{array}$ & $\mathrm{M}=2.64(\mathrm{DT}=.582)$ & $\mathrm{M}=2.46(\mathrm{DT}=.929)$ & $.528(\mathrm{p}=.472)$ \\
\hline \multirow{4}{*}{$\begin{array}{l}\text { Evaluación } \\
\text { UCLA }\end{array}$} & Excelente & $0(0.0 \%)$ & $2(9.1 \%)$ & $\mathrm{p}=.481$ \\
\hline & Bueno & $3(15.8 \%)$ & $2(9.1 \%)$ & \\
\hline & Regular & $4(21.1 \%)$ & $3(13.6 \%)$ & \\
\hline & Malo & $12(44.4 \%)$ & $15(55.6 \%)$ & \\
\hline
\end{tabular}

\section{Discusión y conclusiones}

En este estudio, similar al realizado en otras poblaciones de adultos (Tomas et al., 2008; Zurita et al., 2009) se apreció como la mitad de los participantes analizados seńalaron hacer siempre de una manera continuada actividad física. Es indudable que su práctica genera beneficios a nivel físico y psicológico (disminución de ansiedad, cansancio, estrés,...), influyendo en la prevención de enfermedades, y aportando mejoras en los niveles de autoestima y autoconcepto (Dishman et al., 2006; Babiss y Gangwish, 2009). Del mismo modo Vidarte, Quintero y Herazo (2012) señalan que se produce una mejora notoria de la fuerza, estabilidad y resistencia de tipo aeróbico.

Entendemos que en estas edades la modalidad de práctica no es competitiva y si más relacionada con el bienestar y la calidad de vida, ya que como plantea Zurita (2015), la persona que habitualmente hace deporte tiene niveles de autoestima, autoconcepto y satisfacción incrementados con respecto a los que no hacen, por lo que la actividad física es un elemento clave en la prevención de patologías. Si a esta situación le añadimos que el proceso de envejecimiento varía bastante según el estilo de vida de cada uno(García-Ferrando y Llopis, 2011; Berzosa, Gómez-Angulo, Regodón y Santamaría, 2013; De Souza y Rodrígues, 2014; Martín, Barriopedro, Martínez del Castillo, 
Jiménez-Beattyy Rivero-Herráiz, 2014), el tipo de deporte realizado es más de mantenimiento que de competición.

Entre las tareas que se desarrollan los ejercicios de mantenimiento (andar, carrera suave,...), son los más frecuentes, porque son los más arraigados en poblaciones de personas mayores, ya que son ejercicios poco invasivos y producen un bienestar físico, mental y social considerable entre sus practicantes.

Los sujetos que indican practicar siempre actividad física reportan mayores niveles de resiliencia en todas sus dimensiones que los que no hacen, informan de una mejor salud y un mayor bienestar y que, además, perciben su vida en términos más positivos; que el deporte es el tipo de actividad física que más contribuye en la mejora de esa percepción y que, junto con la edad, la situación laboral y de convivencia, el deporte es una de las cuatro variables que más influyen sobre la percepción de la calidad de vida de la población adulta (Biedma, Fernández-Ballesteros, Martín, Ramos, Rodríguez-Morcillo y Serrano, 2008). Al hilo de esta situación autores como Berzosa et al. (2013), utilizan el caminar como actividad sustitutiva para hacer ejercicio físico con más frecuencia.

Entre los principales predictores de tipo psicosocial del bienestar subjetivo en la adultez tardía se han enfatizado la satisfacción con el estado de salud, las relaciones sociales, la satisfacción de las necesidades básicas, las habilidades personales, la autoeficacia y el ocio activo (Godoy-Izquierdo, Lara, Vázquez, Araque y Godoy, 2012). La práctica de actividad física por parte de los mayores de 65 años ha sido relacionada con una mayor calidad de vida (Rejeski, Brawley y Shumaker, 1996; Sánchez, Ureña y Garcés de los Fayos, 2002; Bohórquez, Lorenzo y García, 2013), por lo que tener una mayor capacidad de resiliencia se encuentra relacionado totalmente con la realización de cualquier tipo de actividad física y no con el sedentarismo.

Se debe partir de la premisa deque en estas edades la modalidad de práctica no es competitiva y si más relacionada con el bienestar y la calidad de vida (García-Ferrando y Llopis, 2011; Berzosa et al., 2013; De Sousa y Rodrígues, 2014; Martín et al., 2014), puesto que el proceso de envejecimiento varía bastante según el estilo de vida de uno y normalmente afecta al desempeño en las actividades de la vida diaria. Cada vez se produce un incremento de la práctica autónoma frente a la organización de las actividades, suponiendo esta situación un cambio en el mercado chileno. Desde otra perspectiva cabe señalar la importancia de que el numeroso grupo de población adulta incorpore la práctica regular de actividad física a sus estilos de vida sea cual sea la tarea realizada.

En este trabajose constatanlos beneficios de tipo psicológico que trae consigo la actividad física y que hace que entre sus practicantes aumenten sus nivelesde resiliencia al igual que acontecía en los estudios de Zurita (2015); Castro-Sánchez et al.(2016); Chacón-Cuberos et al. (2016).

En este trabajo desde el punto de vista de su aplicación práctica presenta un análisis de la influencia que tiene la actividad física en la resiliencia en adultos con problemas de hombro y sirve como indicador para guiar la actuación de estrategias, programas e intervenciones encaminadas a una segunda fase que se está realizando en este momento, consistente en la implementación de ejercicios de reeducación de la articulación del hombro, para evitar la intervención quirúrgica.

En el desarrollo de esta investigación hemos detectado algunas limitaciones como la de un escaso número de participantes y no haber realizado una extensión del estudio a otras poblaciones (española, escolares,....), que nos hubiese dado una mayor perspectiva.

De la investigación realizada las principales conclusiones que se extraen son:

- Aproximadamente la mitad de los adultos realizan actividad física de manera habitual, obteniendo un locus de control y compromiso muy aceptable y valorando en menor medida la espiritualidad.

- No se halló relación entre la práctica de actividad física y el género pero si entre aquellos que desarrollan actividad física de manera continua y las dimensiones de la resiliencia que estaban incrementadas, poniendo de manifiesto que hacer deporte aumenta la resiliencia en los adultos.

\section{Bibliografía}

1. Amstutz, H.C., Sew, A.L., y Clarke, I.C. (1981). UCLA anatomic total shoulder arthroplasty. Clinical Orthopedic, 155, 7-20.

2. Anthony, E.K., Alter, C.F., y Jenson, J.M. (2009). Development of a risk and resilience-based, out-of-school time program for children and youths. Social Work, 54, 45-55.

3. Azpiazu, M., Jentoft, A., Villagrasa, J., Abanades, J., García, N., y Alvear, F. (2002). Factores asociados a mal estado de salud percibido o mala calidad de vida en personas mayores de 65 ańos. Revista Española de Salud Pública, 76, 683-699.

4. Babiss, L. A. y Gangwisch, J. E. (2009). Sports participation as a protective factor against depression and suicidal ideation in adolescents as mediated by self-esteem and social support. Journal of Developmental

and Behavioral Pediatrics, 30(5), 376-384.

5. Berzosa, J., Gómez-Angulo, J., Regodón, C., y Santamaría, L. (2013). ¿Te cuidas? Hábitos de vida de las mujeres de la Comunidad de Madrid. Madrid: Comunidad de Madrid, Consejería de Asuntos Sociales.

6. Biedma, L., Fernández-Ballesteros, R., Martín, M., Ramos, C., Rodríguez-Morcillo, L., y Serrano, R. (2008). Deporte, salud y calidad de vida. Colección Estudios Sociales no 26. Barcelona: Fundación La Caixa.

7. Bohórquez, M. R., Lorenzo, M., y García, A. J. (2013). Felicidad y actividad física en personas mayores. Escritos de Psicología, 6(2), 6-12.

8. Canellas, A. y Rovira, J. (1995). Los hábitos deportivos de la población adulta barcelonesa (15 a 59 años). Apunts: Educación Física y Deportes, 42,75-79. 
9. Connor, K. M. y Davidson, J. R. (2003).Development of a new resilience scale: the Connor-Davidson Resilience Scale (CD-RISC). DepressAnxiety, 18(2), 76-82.

10. De Souza, R. G. y Rodrígues, V. F. (2014). Efectos del entrenamiento de fuerza sobre los niveles de IGF-1 y autonomía funcional de adultos mayores. Revista Ciencias de la Actividad Física UCM, 15(2), 35-42.

11. Dishman, R.K., Hales, D.P., Pfeiffer, K.A., Felton, G., Saunders, R., Ward, D.S., y Pate, R.R. (2006). Physical self-concept and self-esteem mediate cross-sectional relations of physical activity and sport participation with depression symptoms among adolescent girls. HealthPsycho$\log y, 25(3), 396-407$.

12. García-Ferrando, M. y Llopis, R. (2011). Ideal democrático y bienestar personal, Encuesta sobre los hábitos deportivos en España 2010. Madrid: Centro de Investigaciones Sociológicas-Consejo Superior de Deportes.

13. Godoy-Izquierdo, D., Lara, R., Vázquez, M. L., Araque, F. y Godoy, J. F. (2012). Correlates of Happiness Among Older Spanish Institutionalised and NonInstitutionalised Adults. Journal of Happiness Studies, 13(2), 24-52.

14. Gucciardi, D. F., Jackson, B., Coulter, T. J., y Mallett, C. J. (2011). The Connor-Davidson Resilience Scale (CD-RISC): Dimensionality and age-related messurement invariance with Australian cricketers. Psychology of Sport and Exercise, 12, 423-433.

15. Haase, J. E. (2004). The adolescent resilience model as a guide to interventions.Journal of Pediatric Oncology Nursing, 21(5), 289-299.

16. Hicks, J., Epperly, L., y Barnes, K. (2001).Gender, emotional support, and well-being among the rural elderly. Sex Roles, 45, 15-30.

17. Jiménez-Beatty, J. E., Graupera, J. L., Martínez del Castillo, J., Martín, M., y Campos, A. (2007). Motivational factors and physicians' advice in physical activity in the older urban population. Journal of Aging and Physical Activity, 15(3), 241-256.

18. Lamond, A. J., Depp, C.A., Allison,M., Langer, R., Reichstadt, J., y Moore, D. J. (2008). Measurement and predictors of resilience among community-dwelling older woman.JournalPsychiatric Res, 43, 148-154.

19. López-Sánchez, G. F., López-Sánchez, L., y Díaz, A. (2015). Composición corporal y variabilidad de la frecuencia cardiaca: relaciones con edad, sexo, obesidad y actividad física. SPORT TK, 4(2), 33-40.

20. Martín, M., Barriopedro, M.I., Martínez del Castillo, J., JiménezBeatty, J.E., y Rivero-Herráiz, A. (2014). Diferencias de género en los hábitos de actividad física de la población adulta en la Comunidad de Madrid. Revista Internacional de Ciencias del Deporte, 38(10), 319-335.

21. Martínez, J., Jiménez-Beatty, J. E., González, M. D., Graupera, J. L.,
Martín, M., Campos, A., y Del Hierro, D. (2009). Los hábitos de actividad física de las mujeres mayores en Espańa. RevistaInternacional de CienciasdelDeporte,

22. Peixoto, G., Fiquetti, P., Polisello, N., Barbosa, G., Ejnisman, B., y Cohen, M. (2013).Glenohumeral range of motion in handball players with and without throwing-related shoulder pain.Journal Shoulder Elbow Surg, 22, 602-607.

23. Pinguart, M., y Sorensen, S. (2001). Gender differences in self-concept and psychological well-being in old age: A meta-analysis. Journal of Gerontology, 56, 195-214.

24. Rejeski, W. J., Brawley, L. R., y Shumaker, S. A. (1996).Physical activity and healthrelated quality of life.Exercise and SportsSciencesReviews, 24, 71-108.

25. Rioseco, R., Quezada, M., Ducci, M., y Torres, M. (2008). Cambio en las redes sociales de adultos mayores beneficiarios de programas de vivienda social en Chile. Revista Panamericana de Salud Pública, 23, 147-153.

26. Sánchez, P. A., Ureña, F., y Garcés de los Fayos, E. J. (2002). Repercusiones de un programa de actividad física gerontológica sobre la aptitud física, autoestima, depresión y afectividad. Cuadernos de Psicología del Deporte, 2(2), 57-73.

27. Subirats, E., Subirats, G., y Soteras, I. (2012). Prescripción de ejercicio físico: indicaciones, posología y efectos adversos. Medicina Clinica, 138(1), 18-24

28. Tomás, M., Meléndez, J., y Navarro, E. (2008). Modelos factoriales confirmatorios de las escalas de Ryff en una muestra de personas mayores. Psicothema, 20, 304-310.

29. Vidarte, J.A., Quintero, M.V., y Herazo, Y. (2012). Efectos del ejercicio físico en la condición física funcional y la estabilidad en adultos mayores. Hacia la promoción de la salud, 17(2), 79-90.

30. Vinson, J. (2008). Childrenwithasthma: Initialdevelopment of thechildresiliencemodel. PediatricNursing,28 (2), 149-158.

31. Vivaldi, F. y Barra, E. (2012). Bienestar Psicológico, Apoyo Social Percibido y Percepción de Salud en Adultos Mayores. Terapia Psicológica, 30(2), 23-29.

32. Zurita, F. (2015). Influencia de los factores psicológicos sobre las lesiones deportivas en deportes de equipo. Tesis Doctoral: Universidad de Jaén.

33. Zurita, F., Fernández, R., Cepero, M., Zagalaz, M.L., Valverde, M., y Ramírez, P. (2009). The relationship between pain and physical activity in older adults that begin a program of physical activity, Journal of $\mathrm{Hu}$ man and Sport, 4(3), 284-297. 